\title{
Barriers to involvement of Chinese demographers in climate change research
}

\section{Xizhe Peng and Qin Zhu*}

The population and its development trends are important topics in the fields of climate change mitigation and adaptation. Although academic communities (like the IUSSP) have encouraged them to do so, few demographers have devoted themselves to research on climate change.

In China, only a handful of demographers are engaged in climate change research. According to our review of literature published in Chinese core journals in the past decade (2005-2014), there are 17,210 papers with the keyword 'climate change', 'carbon emissions', or 'emission of $\mathrm{CO}_{2}$ '. Of these papers, only $254(1.5 \%)$ also had the keyword 'population' or 'demography', and only $41(0.2 \%)$ are classified as population studies.

Why are so few demographers focusing on climate change research? Within the context of China, we will discuss the knowledge structure and research methods of demographers, the incentive system for scientific research, the population development trends, and the relationship between population and climate change.

First, narrowly defined, the focus of demography is on theories and analytical methods of population change, including fertility, mortality, and the spatial distribution of the population. The field of climate change involves much wider horizons with grand narratives that go beyond the core demographic themes, such as industrial development, energy utilization, ocean and land coverage, and environmental change. It is difficult to use only demographic techniques when examining complicated climate change issues. At the same time, the demographic factors considered in climate change studies are generally represented by a single variable (the change in population size), and even this variable is often treated as exogenous by researchers from other disciplines. This narrow perspective tends to discourage demographers' participation in such studies. In addition, many of the individuals who have received professional training in demography also work as

\footnotetext{
* Xizhe Peng (corresponding author), Research Center for Population and Development Policies, Fudan University, Shanghai, China

Email: xzpeng@fudan.edu.cn
}

Qin Zhu, Research Center for Population and Development Policies, Fudan University, Shanghai, China 
economists or sociologists, and thus make use of theories and methods of economics and sociology, as well as those of demography. As these disciplinary boundaries are not always clear, some of the demographic studies on climate change may not have been classified as demographic.

Second, the existing funding system tends to "value natural sciences but undervalue social sciences'. As in China demography is considered a social science discipline, demographers are often at a disadvantage when applying for funding for climate change studies, which are dominated by natural scientists. Furthermore, the actual funding of individual projects for climate change is much lower if the funding source distributes funds for social science rather than 'hard science' projects, even if they have similar designs and technical components. This is true in general not only of demographic studies, but also of studies in most other social science disciplines. This situation has existed over the past few decades, mainly due to both the government's priorities and the market demand for research on the natural sciences and applied technologies. These scientific research incentive mechanisms have not only affected the enthusiasm of demographers for climate change research; they have further widened the disparities in research opportunities and quality between the social sciences (such as demography) and the natural sciences. This lack of funding and of governmental support may negatively affect the ability of demographers to take the lead in the field of climate change research.

Third, the development of demography in China as an academic discipline has been largely associated with the effort to control population that has played a central role in China's modernization process. It is therefore understandable that fertility-related research, including studies on family planning programs, have long dominated the research agenda. China's strong economic growth over the past three decades has, however, greatly reduced the public's concerns about the Earth's carrying capacity and 'the Malthusian trap', and has changed people's understanding of the relationship between population and pressure on resources and the environment. Chinese society is experiencing a profound social transformation. Today, Chinese demographers are overwhelmingly occupied with researching large-scale migration, rapid urbanization, and other emerging population issues, such as future labor shortages and aging. Demographers' capacity to focus on the issue of climate change is certainly limited by demands that they address these new and emerging population-related challenges. Moreover, while public awareness of climate change is rising in China, it is often considered as a medium- to long-term issue.

Last but not least, the characteristics of climate change mitigation and adaptation limit the role demographers can play in this research field. In terms of climate change mitigation, demographic factors mainly have indirect effects. For example, while changes in the population may be expected to lead to changes in patterns of production and consumption, these kinds of shifts, and the related benefits to and cooperation between countries, are research topics that are typically addressed by economists and political scientists. In terms of climate change adaptation, demographic factors can also be indirectly reflected through ecological protection, population migration, disaster prevention, and other human behaviors. 
The contributions of ecologists, disaster prevention specialists, and geographers appear to be more relevant in these research fields, leaving demographers with no particular comparative advantages.

While a range of factors that are likely to discourage demographers from engaging in research on the link between population and climate change have been discussed here, this does not mean that demographers have no contribution to make to this field. Indeed, as more demographers are highlighting the role of demography in climate change, this relationship is increasingly being recognized. Chinese demographers have made significant contributions to China's climate change research and to the formulation and implementation of relevant government policies. Journal articles written by demographers are widely cited by climate change researchers, although the number of such articles is relatively small. Through the joint efforts of Chinese demographers and scientists in other disciplines, a discipline known as 'population, resources, and environmental economics' has been formally established by China's education authority, and degree programs have been set up in more than a hundred Chinese universities. Students who are enrolled in this discipline come from a variety of academic backgrounds, and population study is a core component of these programs. An interdisciplinary academic journal, 'China Population, Resources and Environment', has published articles in the field of population and climate change. It is expected that the younger generation of demographers in China will become increasingly involved in climate change research. The population studies community should provide better guidance to young demographers, and develop more collaborations with other disciplinary networks. These efforts will not only enrich our scientific knowledge on climate change; they will promote the development of demography itself. 
